\title{
rs2476601 polymorphism in PTPN22 is associated with Crohn's disease but not with ulcerative colitis: a meta-analysis of 16,838 cases and 13,356 controls
}

\author{
Abdellah Hedjoudje ${ }^{a, b}$, Chérifa Cheurfa ${ }^{b, c}$, Clément Briquez ${ }^{a}$, Allen Zhang ${ }^{d}$, Stéphane Koch ${ }^{a}$, \\ Lucine Vuitton ${ }^{\mathrm{a}}$ \\ Centre Hospitalier Régional Universitaire de Besançon, Besançon; Université Paris Descartes, Paris, France; CHU \\ Charles Nicolle, Rouen, France; Johns Hopkins University, Baltimore, United States
}

\section{Abstract}

${ }^{\mathrm{a}}$ Gastro-entérologie, Centre Hospitalier Régional Universitaire de Besançon, Besançon (Abdellah Hedjoudje, Clément Briquez, Stéphane Koch, Lucine Vuitton); 'Faculté de Médecine, Université Paris Descartes, Paris (Chérifa Cheurfa); ${ }^{\mathrm{A} A n e ́ s t h e ́ s i e ~ r e ́ a n i m a t i o n, ~} \mathrm{CHU}$ Charles Nicolle, Rouen, France (Chérifa Cheurfa); `Johns Hopkins University Evidence-based Practice Center, Johns Hopkins University, Baltimore, United States (Allen Zhang)

Conflict of Interest: None

Correspondence to: Abdellah Hedjoudje, Centre Hospitalier Régional Universitaire Jean Minjoz Service de Gastro-entérologie, Bâtiment Gris 1 rue Alexandre Fleming 25000 Besançon, France, e-mail: abdellah. hedjoudje@gmail.com

Received 24 October 2016; accepted 07 December 2016; published online 5 January 2017

DOI: https://doi.org/10.20524/aog.2017.0121

\section{Introduction}

Inflammatory bowel diseases (IBD), consisting of ulcerative colitis (UC) and Crohn's disease (CD), are chronic inflammatory disorders of the gut that are probably the result of a dysregulated immune response to the gut microflora in genetically predisposed individuals, triggered by environment $[1,2]$. The annual incidence of UC varies from $0-19.2$ per 100,000 in North America and from 0.6-24.3 per 100,000 in Europe, corresponding to a prevalence of $37.5-248.6$ per 100,000 and 4.9-505 per 100,000 , respectively. The incidence of CD is similar in western countries (0-20.2 per 100,000 in North America; 0.3-12.7 per 100,000 in Europe). The incidence and prevalence of IBD are increasing over time and in different regions around the world [3]. IBD are polygenic diseases for 
which up to 163 genes have been found to increase the risk of susceptibility [4-6]. Previous reports indicated several single nucleotide polymorphisms (SNPs) in different regions of the genome increasing risk of IBD. One of the most important non-human leukocyte antigen (HLA) common susceptibility alleles for autoimmunity is the $1858 \mathrm{C} / \mathrm{T}$ SNP of protein tyrosine phosphatase non-receptor 22 (PTPN22) (rs2476601).

The PTPN22 gene encodes a protein tyrosine phosphatase, non-receptor type 22 (PTPN22), located on chromosome 1p13. This $110-\mathrm{kDa}$ lymphoid-specific phosphatase (Lyp) plays a critical role as a negative regulator of T-cell activation by dephosphorylating T-cell receptor activation dependent kinases (Csk kinase) [7], expressed exclusively in immune cells; therefore, this gene may be associated with autoimmunity [8].

SNPs within the PTPN22 gene may affect the regulatory role of the Lyp. A non-synonymous SNP at position 1858 of PTPN22 gene (rs2476601) changes the amino acid from an arginine to a tryptophan and affects the ability of Lyp to interact with the Csk kinase, thus avoiding the formation of the complex and the resulting suppression of T-cell activation. In vitro experiments have shown that the PTPN22 1858T allelic variant binds less efficiently to Csk than does the $\mathrm{C}$ allele. This suggests that individuals lacking the $\mathrm{C}$ allele of PTPN22 may have a reduced capacity to downregulate T-cell responses and may therefore be more susceptible to autoimmunity. Several studies have investigated a potential association of PTPN22 C1858T polymorphism with various autoimmune disorders, including rheumatoid arthritis [9], systemic lupus erythematosus [10], Grave's disease [11], and type 1 diabetes mellitus [12]. However, contradictory results have been published regarding the association of rs 2476601 variant with either $\mathrm{CD}$ or UC. This inconsistency might be due to differences in sample size, patient ethnicity, or allele frequencies. Accordingly, given these results, we performed a meta-analysis to determine quantitatively the risk of CD and UC with the rs2476601 variant under an allelic, recessive, dominant and co-dominant model.

\section{Materials and methods}

\section{Selection of eligible studies}

This meta-analysis followed the Preferred Reporting Items for Systematic Reviews and Meta-analysis (PRISMA) criteria [13]. The eligible studies were obtained by searching online databases, MEDLINE and EMBASE. The following keywords were used for searching: ("inflammatory bowel disease" OR "Crohn's disease" OR "ulcerative colitis") AND ("polymorphism* "OR "variant") AND ("protein tyrosine phosphatase nonreceptor" OR "PTPN22" OR "lymphoid protein tyrosine phosphatase"). The most recent research was performed on April 30th 2016, and there was no limitation on the research. The references of reviews and retrieved articles were also searched simultaneously to find additional eligible studies. Research was performed using the Entrez interface of the National Center for Biotechnology Information (ncbi) from the terminal of a Linux station (ubuntu 14.04.2) and through a graphical interface in EMBASE.

\section{Data extraction}

Relevant information from the articles were extracted from the studies independently by the first (AH) and second (CC) authors. Disagreements were resolved through discussion to reach consensus or were adjudicated by a third author. If genotype information was lacking, we tried to contact the corresponding authors in order to obtain the required data. If they did not provide data, those studies were excluded from our review. General characteristics (e.g. demography, genotyping method) of the included studies were extracted. We extracted the following information from each included study: first author's name, publication year, sample size, source of controls, ethnicity, genotyping method, matching variables of controls with cases, age, sex distribution, and counts of alleles and genotypes in both cases and controls. We restricted our metaanalysis to candidate gene studies. The included studies had to meet the following criteria: 1) they concerned the association between rs2476601 and IBD; 2) they were case-control design or cohort studies; 3 ) they reported genotype frequencies or allele frequencies of the rs 2476601 polymorphism. The exclusion criteria were: 1) review-articles, case reports and comments; and 2) duplicate publications.

\section{Statistical analysis}

The Hardy-Weinberg equilibrium (HWE) was examined in control groups using Fisher's exact test. If the study was found not to be in HWE with a P value less than 0.05 , it was considered to be disequilibrium. Allele frequencies of the PTPN22 rs2476601 polymorphisms in each of the studies were determined using the allele counting method. Allelic effect contrast was examined for the minor allele vs. the common allele. The genetic models evaluated for pooled ORs included allelic contrast ( $\mathrm{C}$ vs. T), recessive models (CC vs. CT + TT), dominant models (CC + CT vs. TT), and the homozygote model (CC vs. TT). To evaluate the strength of association, the pooled ORs and their 95\%CIs were determined for each study, and within- and between-study heterogeneity was assessed using Cochran's $Q$ statistic [14]. The heterogeneity test was used to assess the probability of the null hypothesis that all studies were evaluating the same effect. The randomeffects model was used for meta-analysis when a significant $Q$ statistic $(\mathrm{P}<0.10)$ indicated heterogeneity across studies, while the fixed-effect model was used when heterogeneity was not indicated. The fixed-effect model assumes that genetic factors have similar effects on disease susceptibility across all studies and that observed variations between studies are caused by chance alone [15]. The random-effect model assumes that studies show substantial diversity, and assesses both withinstudy sampling errors and between-study variances [16]. When study groups are homogenous, the two models are similar, but if this is not the case, the random effects model 
usually provides wider CIs than the fixed effects model. The random effects model is best used in the presence of significant between-study heterogeneity [16]. We quantified the effect of heterogeneity by using the recently developed $\mathrm{I}^{2}$ measure, where $\mathrm{I}^{2}=100 \% \times(\mathrm{Q}-\mathrm{df}) / \mathrm{Q}$, where $\mathrm{df}$ is degrees of freedom [17]. The $\mathrm{I}^{2}$ measure ranges between $0 \%$ and $100 \%$ and represents the proportion of inter-study variability attributable to heterogeneity rather than chance. $\mathrm{I}^{2}$ values of $25 \%, 50 \%$, and $75 \%$ were defined as low, moderate, and high estimates, respectively. All statistical analyses were performed using the general package for meta-analysis (version 4.6-0, depends on $\mathrm{R} \geq 2.9 .1$ ).

\section{Evaluation of publication bias}

Finally, potential publication bias was assessed using a funnel plot and Egger's test, which measures funnel plot asymmetry on a natural logarithm scale of ORs [18]. Funnel plot should be interpreted with caution, as they usually require a large number of studies.

\section{Results}

\section{Search strategy}

According to the search strategy (Fig. 1), 34 studies were initially included; 15 papers were excluded after reading the title and abstract, 1 paper was not relevant to rs2476601, while 1 paper [19] included three different populations, which were treated as three different studies. Two studies $[20,21]$ that presented a significant deviation from Hardy-Weinberg equilibrium in controls $(\mathrm{P}=0.041$ and $\mathrm{P}=0.010)$ were excluded from the analysis (Table 1). One article [22] was excluded on the basis of a T recessive test, as the TT genotype was not reported in the population of interest.

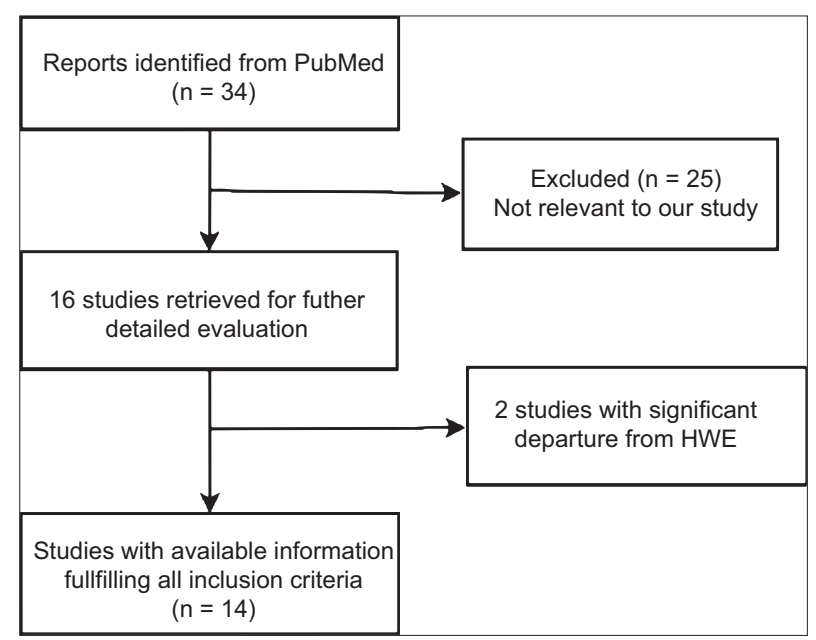

Figure 1 Flow chart showing selection of studies and specific reasons for exclusion from the study
A total of 14 studies [9,23-33] were thus included in the analysis (Tables 2 and 3) involving a total of 13,356 controls, 8182 patients with CD, and 8656 with UC. The methodological quality of the studies were also evaluated (Table 4).

\section{Quantitative analysis of PTPN22 rs2476601 and CD}

They were 14 studies investigating the association between PTPN22 and CD, which included a total of 13,370 patients and 16,888 controls (Table 2). The presence of the $\mathrm{C}$ allele significantly increased the risk of having CDs (OR 1.28, 95\%CI 1.17-1.40). We could not find significant heterogeneity between the studies $\left(\mathrm{I}^{2}=37.5 \%, \mathrm{P}=0.0835\right)$. The Funnel plot showed no asymmetry.

The CC genotype was compared with CT and TT assuming a recessive model for the $\mathrm{C}$ allele. The pooled $\mathrm{OR}$ was 1.34 (95\%CI 1.20-1.49) and no significant between-study heterogeneity was found $\left(\mathrm{I}^{2}=3.5 \%, \mathrm{P}=0.4077\right)$ (Fig. 2). Assuming a dominant model for $\mathrm{C}$ allele, we could not find a significant risk for having $\mathrm{CD}$ (OR 1.14, 95\%CI 0.72-1.8). The between-study heterogeneity was low $\left(\mathrm{I}^{2}=14.5 \%, \mathrm{P}=0.3094\right)$ (Fig. 2, Table 4).

\section{Quantitative analysis of PTPN22 rs2476601 and UC}

Seven studies investigated the allelic risk of rs2476601, including a total number of 6971 patients with UC and 9715 controls. The results are summarized in a forest plot (Fig. 3). No significant association was found (OR 1.06, 05\%CI 0.98-1.14), assuming a fixed effect model because of a low between-study heterogeneity $\left(\mathrm{I}^{2}=32.2 \%, \mathrm{P}=0.1064\right)$. No association was found assuming a dominant effect for C allele (OR 0.92, 95\%CI 0.641.31 ), or a recessive model for $\mathrm{C}$ allele: OR $1.09,95 \% \mathrm{CI} 0.97$ 1.22 , and OR $1.11,95 \%$ CI $0.94-1.31$, assuming a fixed effect and a random effect model respectively $\left(\mathrm{I}^{2}=50 \%, \mathrm{P}=0.0639\right)$. In addition, measuring the risk of carrying CC compared to TT allele on a co-dominant model, we could not find a significant increased risk of UC with either genotype $(\mathrm{OR}=0.84,05 \% \mathrm{CI}$ 0.55-1.28) assuming a fixed effect model.

\section{Sensitivity analysis}

We performed a sensitivity analysis for statistically significant results. For the association of the PTPN22 rs2476601 polymorphism and CD or UC susceptibility among the overall populations, the observed significant result was not materially altered by sequentially excluding each study.

\section{Publication bias}

Egger's test indicated no significant publication bias in the allelic, dominant, recessive and co-dominant models for $\mathrm{CD}$ $(\mathrm{P}=0.48, \mathrm{P}=0.75, \mathrm{P}=0.78$ and $\mathrm{P}=0.48$, respectively). There was 
Table 1 Hardy-Weinberg Equilibrium (HWE) deviation P-values in controls and cases

\begin{tabular}{|c|c|c|c|c|c|c|c|}
\hline Author & Year & Ethnicity & Phenotype & $\mathrm{CC}$ & $\mathrm{CT}$ & TT & HWE-P-values \\
\hline Bank & 2014 & Danish & Controls & 588 & 166 & 11 & 0.123 \\
\hline Bank & 2014 & Danish & $\mathrm{CD}$ & 533 & 85 & 2 & 0.072 \\
\hline Bank & 2014 & Danish & UC & 338 & 68 & 4 & 0.093 \\
\hline Bank & 2014 & Danish & IBD & 871 & 153 & 6 & 0.080 \\
\hline Chen & 2013 & Han Chinese & Controls & 300 & 0 & 0 & $\mathrm{NC}$ \\
\hline Chen & 2013 & Han Chinese & UC & 165 & 0 & 0 & NC \\
\hline Diaz & 2011 & Spanish & Controls & 1467 & 209 & 9 & 0.067 \\
\hline Diaz & 2011 & Dutch & Controls & 683 & 142 & 9 & 0.096 \\
\hline Diaz & 2011 & New Zealander & Controls & 454 & 106 & 3 & 0.099 \\
\hline Diaz & 2011 & Spanish & $\mathrm{CD}$ & 626 & 69 & 4 & 0.055 \\
\hline Diaz & 2011 & Dutch & $\mathrm{CD}$ & 575 & 94 & 3 & 0.074 \\
\hline Diaz & 2011 & New Zealander & $\mathrm{CD}$ & 414 & 60 & 3 & 0.069 \\
\hline Diaz & 2011 & Spanish & UC & 571 & 81 & 6 & 0.071 \\
\hline Diaz & 2011 & Dutch & UC & 468 & 67 & 4 & 0.070 \\
\hline Diaz & 2011 & New Zealander & UC & 366 & 76 & 6 & 0.098 \\
\hline Diaz & 2011 & Spanish & IBD & 1197 & 150 & 10 & 0.063 \\
\hline Diaz & 2011 & Dutch & IBD & 1043 & 161 & 7 & 0.072 \\
\hline Diaz & 2011 & New Zealander & IBD & 780 & 136 & 9 & 0.083 \\
\hline Hradsky & 2008 & Czech & controls & 398 & 100 & 3 & 0.106 \\
\hline Hradsky & 2008 & Czech & $\mathrm{CD}$ & 275 & 66 & 4 & 0.107 \\
\hline Latiano & 2007 & Italian & Controls & 235 & 21 & 0 & 0.041 \\
\hline Latiano & 2007 & Italian & $\mathrm{CD}$ & 283 & 18 & 0 & 0.030 \\
\hline Latiano & 2007 & Italian & UC & 278 & 28 & 0 & 0.046 \\
\hline Latiano & 2007 & Italian & IBD & 561 & 46 & 0 & 0.038 \\
\hline Martin & 2005 & Spanish & Controls & 714 & 95 & 3 & 0.062 \\
\hline Martin & 2005 & Spanish & Controls & 714 & 95 & 3 & 0.062 \\
\hline Martin & 2005 & Spanish & UC & 474 & 67 & 3 & 0.067 \\
\hline Martin & 2005 & Spanish & $\mathrm{CD}$ & 514 & 51 & 4 & 0.052 \\
\hline Morgan & 2010 & New Zealander & Controls & 379 & 85 & 8 & 0.107 \\
\hline Morgan & 2010 & New Zealander & $\mathrm{CD}$ & 260 & 52 & 3 & 0.092 \\
\hline Prescott & 2005 & British & Controls & 312 & 61 & 1 & 0.084 \\
\hline Prescott & 2005 & British & Controls & 312 & 61 & 1 & 0.084 \\
\hline Prescott & 2005 & British & Controls & 312 & 61 & 1 & 0.084 \\
\hline Prescott & 2005 & British & $\mathrm{CD}$ & 254 & 37 & 3 & 0.073 \\
\hline Prescott & 2005 & British & $\mathrm{CD}$ & 254 & 37 & 3 & 0.073 \\
\hline Prescott & 2005 & British & UC & 192 & 26 & 2 & 0.068 \\
\hline Sfar & 2010 & Tunisian & Controls & 98 & 2 & 0 & 0.010 \\
\hline Sfar & 2010 & Tunisian & $\mathrm{CD}$ & 68 & 37 & 0 & 0.176 \\
\hline Sfar & 2010 & Tunisian & $\mathrm{UC}$ & 46 & 13 & 0 & 0.110 \\
\hline Sfar & 2010 & Tunisian & IBD & 114 & 50 & 0 & 0.152 \\
\hline Skieceviciene & 2013 & Lithuanian & Controls & 827 & 269 & 25 & 0.142 \\
\hline Skieceviciene & 2013 & Lithuanian & UC & 308 & 117 & 11 & 0.159 \\
\hline Wagenleiter & 2005 & German & Controls & 203 & 49 & 2 & 0.104 \\
\hline Wagenleiter & 2005 & German & $\mathrm{CD}$ & 122 & 23 & 1 & 0.086 \\
\hline
\end{tabular}

$\overline{C D}$, Crohn's disease; IBD, inflammatory bowel disease; UC, ulcerative colitis 


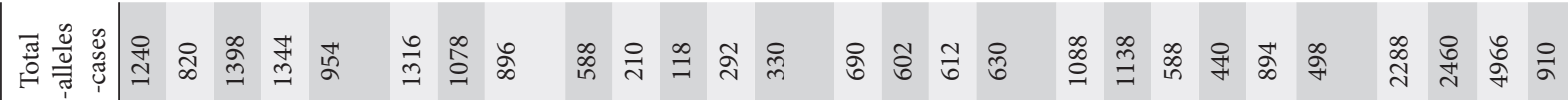

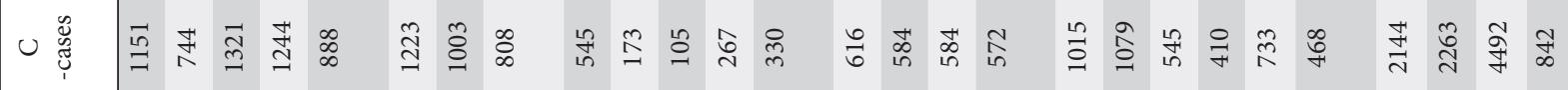

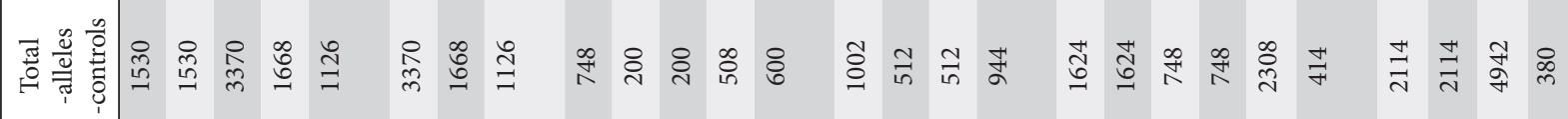

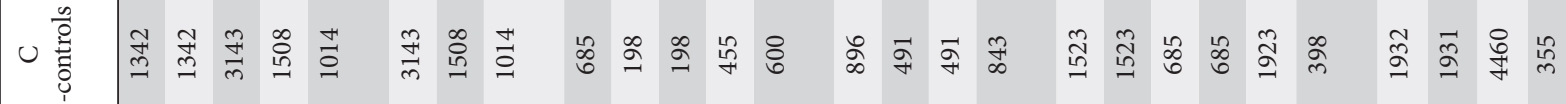
E

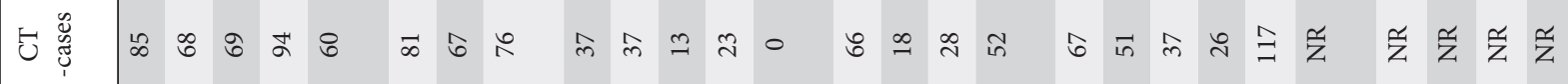

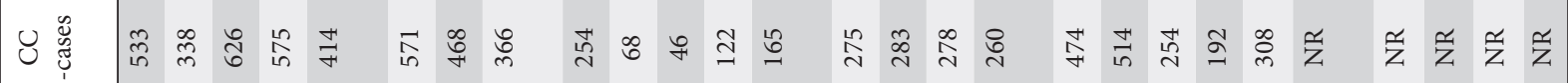
苗酸

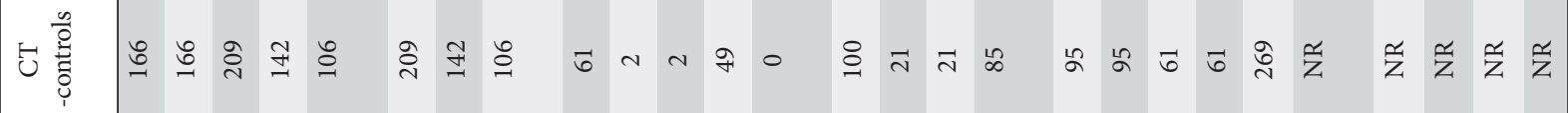

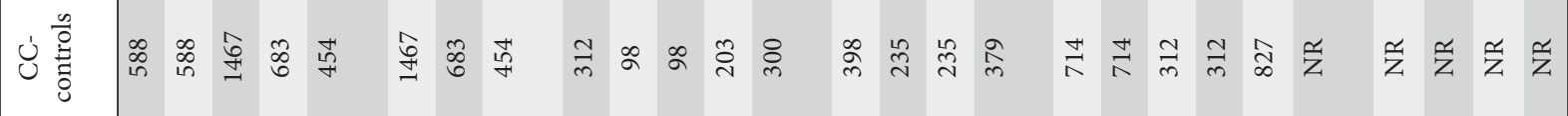

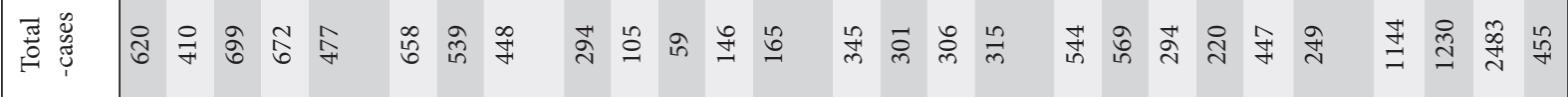

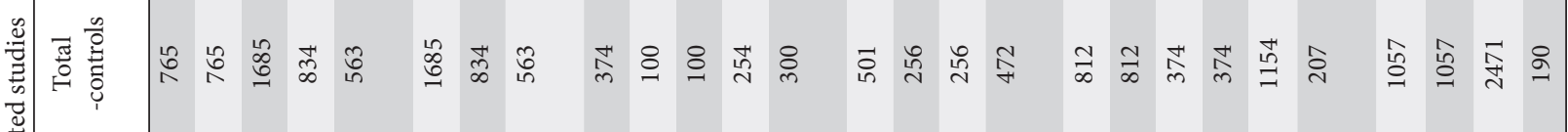

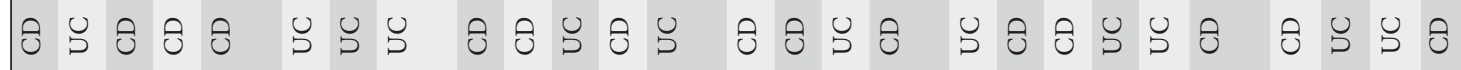

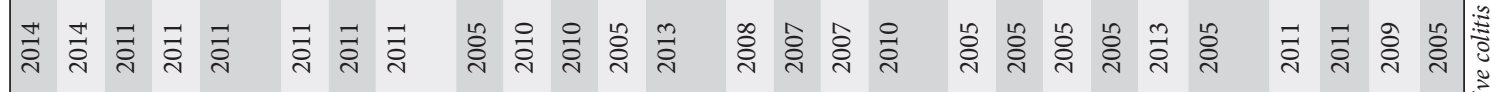

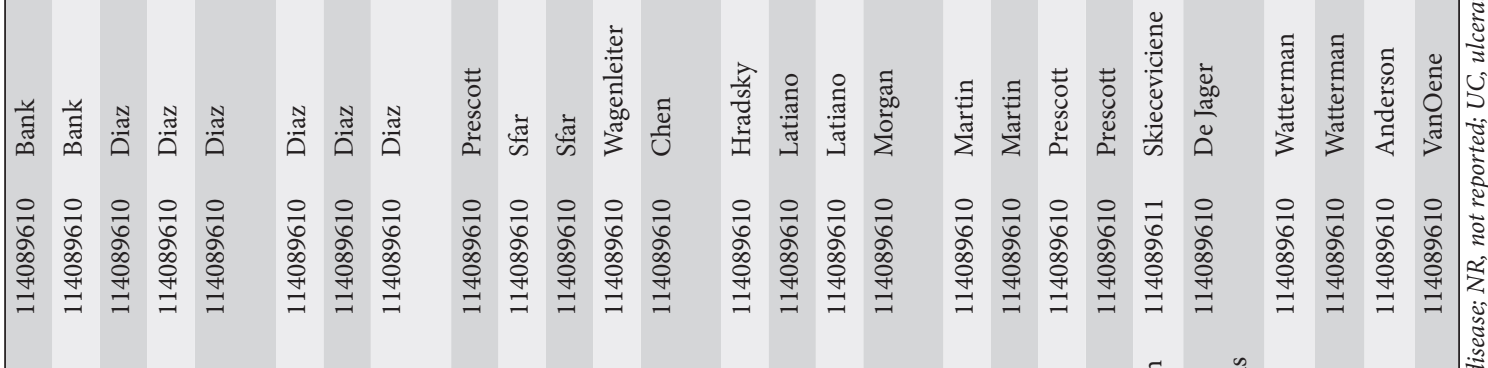

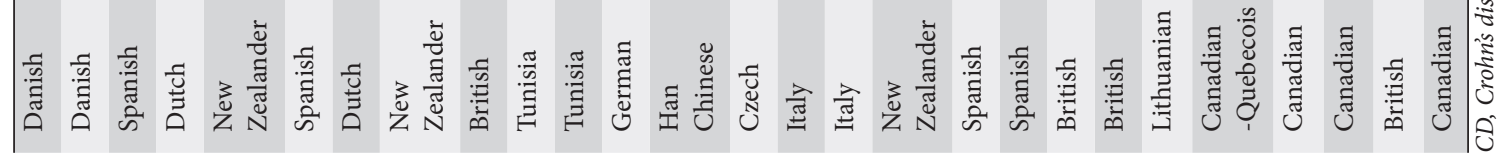


Table 3 Demographic and baseline characteristics of the selected studies

\begin{tabular}{|c|c|c|c|c|c|c|c|c|c|}
\hline \multirow[t]{2}{*}{ Study-year } & \multirow[t]{2}{*}{ Method } & \multirow[t]{2}{*}{ Ethnicity } & \multicolumn{2}{|l|}{ Controls } & \multicolumn{2}{|l|}{ UC } & \multicolumn{2}{|l|}{$\mathrm{CD}$} & \multirow{2}{*}{$\begin{array}{l}\text { Quality } \\
\text { of the } \\
\text { study }\end{array}$} \\
\hline & & & Mean & $\begin{array}{c}\text { Male } \\
(\%)\end{array}$ & Mean Age & $\begin{array}{c}\text { Male } \\
(\%)\end{array}$ & Mean & $\begin{array}{c}\text { Male } \\
(\%)\end{array}$ & \\
\hline \multirow[t]{2}{*}{ Bank, 2014} & Kasp & Danish & 43 (median) & 52 & 42 (median) & 49 & 37 (median) & 44 & 9 \\
\hline & TaqMan & Spanish & NR & NR & NR & NR & NR & NR & \\
\hline \multirow[t]{2}{*}{ Diaz, 2011} & TaqMan & Dutch & NR & NR & NR & NR & NR & NR & 9 \\
\hline & TaqMan & New Zealander & NR & NR & NR & NR & NR & NR & \\
\hline Prescott, 2005 & TaqMan & British & NR & NR & NR & NR & NR & NR & 2 \\
\hline Sfar, 2010 & RFLP-PCR & Tunisian & NR & 52 & 38 & 30 & 36 & 52 & 9 \\
\hline Wagenleiter, 2005 & RFLP-PCR & German & 38 & $72 \%$ & NR & NR & NR & NR & 8 \\
\hline Chen, 2013 & RFLP-PCR & Han Chinese & NS & NS & 41.3 & 57.6 & 43.7 & 55 & 11 \\
\hline Hradsky, 2008 & TaqMan & Czech & 20.27 (median) & $64 \%$ & NS & NS & 21 & 44 & 11 \\
\hline Latiano, 2007 & TaqMan & Italian & NR & NR & 35 & $52 \%$ & 35 & 57 & 10 \\
\hline Morgan,2010 & MALDI-TOF & New Zealander & NR & 66.5 & NS & NS & NR & 31.4 & 11 \\
\hline Martin, 2005 & TaqMan & Spanish & NR & NR & NR & NR & NR & NR & 9 \\
\hline Skieceviciene, 2013 & TaqMan & Lithuanian & 40.2 & NR & 44.4 & NR & NS & NS & 10 \\
\hline De Jager, 2005 & SeqenomMassArray & Canadian-Quebecois & NR & NR & NS & NS & NR & NR & 6 \\
\hline Watterman, 2011 & IlluminaGoldenGate & Canadian & NR & 35.8 & NR & 49.2 & NR & 52.7 & 9 \\
\hline Criswell, 2005 & PSQHS96APyrosequencer & American & NR & NR & NR & NR & NR & NR & 8 \\
\hline Anderson, 2009 & iPLEX & British & NR & NR & NR & $50 \%$ & NR & $39 \%$ & 11 \\
\hline Van Oene, 2005 & MALDITOF & Canadian & NR & NR & NS & NS & NR & NR & 9 \\
\hline
\end{tabular}

NR, not reported; NS, not studied; $C D$, Crohn's disease; UC, ulcerative colitis

also no significant publication bias in the allelic, dominant, recessive and co-dominant models for $\mathrm{UC}(\mathrm{P}=0.49, \mathrm{P}=0.6$, $\mathrm{P}=0.91$ and $\mathrm{P}=0.49$, respectively). The funnel plots were symmetrical for all tests.

\section{Discussion}

The incidence of IBD is increasing worldwide and the rising prevalence of immune diseases is thought to be influenced by genetic and environmental factors [1,2]. PTPN22 encodes the gatekeeper of T-cell receptor signaling, cytoplasmic Lyp and as such is a likely candidate risk factor for UC because of its role in down-regulation of T-cell activity [34].

So far, genome-wide association studies have found more than 163 genes that are associated with IBD in a Caucasian population from. Of these genes, 110 have been shown to be significantly associated with both UC and CD, but 23 showed risk effects that were UC specific and 30 were CD-only loci [5]. This suggests that there are different biological pathways in these two diseases that are well reflected by the different phenotypes and still need to be explored. There have been conflicting results, with some studies showing an increased risk associated with the T allele [19], whereas other studies, such as the one conducted by Silverberg et al [35], did not report any significantly increased risk. Most studies have failed to show a significant association and this could be due to a lack of power. A meta-analysis is a powerful tool that can improve the statistical performance by combining the results of multiple studies. Therefore, in the present study, we investigated whether the PTPN22 1858C/T polymorphism contributes to IBD susceptibility, using a meta-analysis approach.

We pooled data from 14 individual studies results to determine the effect of a non-synonymous SNP at position 1858 of the PTPN22 gene (rs2476601) on CD and UC, This is the first meta-analysis of candidate gene studies exploring the variant rs2476601 of PTPN22. We determined that individuals carrying minor allele $C$ in rs2476601 have a greater risk of developing CD (OR 1.28, 95\%CI 1.17-1.40) relative to those carrying the $\mathrm{T}$ allele, while carrying the $\mathrm{C}$ allele does not significantly change the risk of developing UC. Genotypic effects were also estimated for PTPN22 and the risk of CD was greater (OR 1.34, 95\%CI 1.20-1.49) with CC genotype compared to CT or TT. In our study we did not detect significant between-study heterogeneity among the case-control studies included. Interestingly, the T allele was associated with a lower risk of $\mathrm{CD}$, but an increased risk for other immune diseases, such as type 1 diabetes mellitus, or rheumatoid arthritis [10,12].

The rs2476601 polymorphism disrupts the interaction between Lck and Lyp, leading to reduced phosphorylation of Lyp, which ultimately contributes to gain-of-function 
rs2476601 polymorphism in PTPN22 7

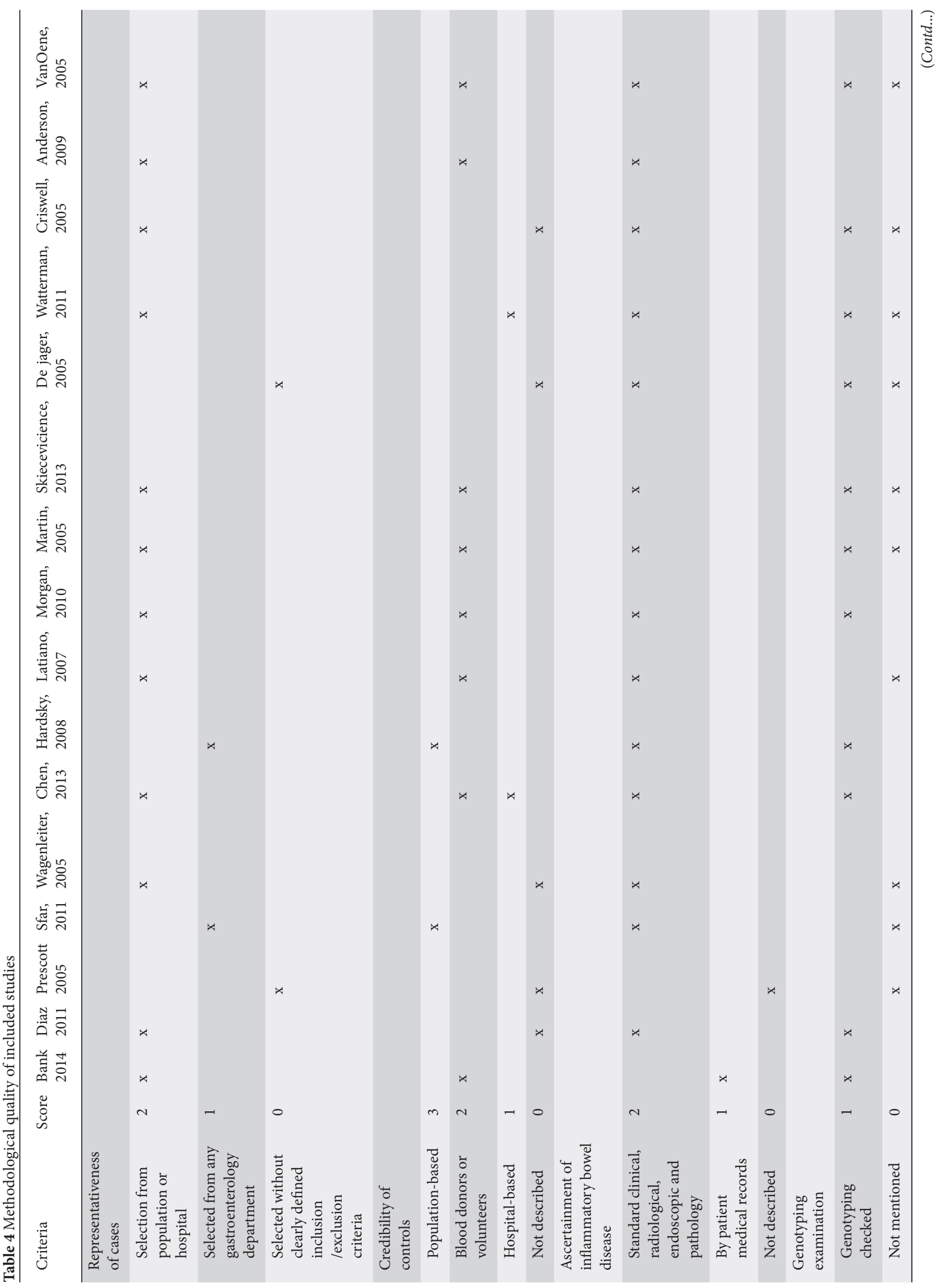




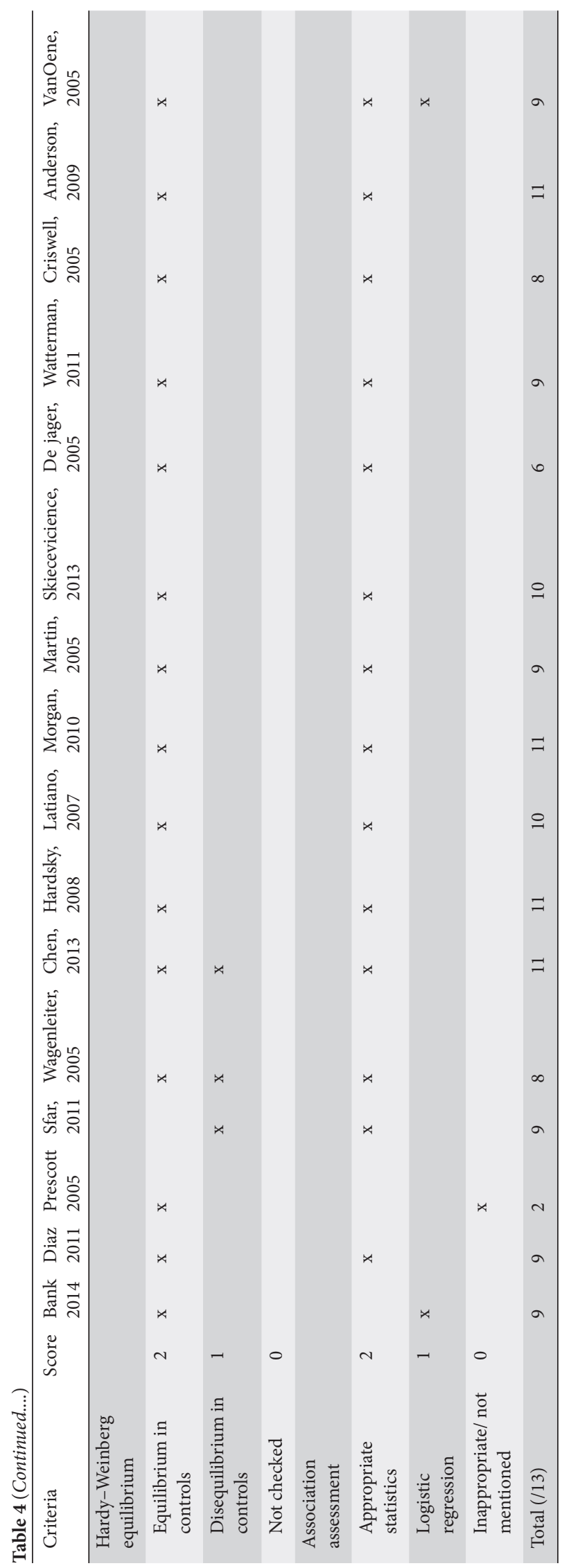

inhibition of T-cell activation [36]. Despite the promising role for PTPN22 mutation in the risk of UC, we were unable to find a significant association in our meta-analysis. Interestingly, other polymorphisms, such as $-1123 \mathrm{G} / \mathrm{C}$, located in the promoter region of PTPN22 have been shown to be correlated with other autoimmune diseases $[37,38]$. Another polymorphism, $+788 \mathrm{G} / \mathrm{A}$ in exon 10 of PTPN22, has been found to be associated with UC [22] in a Chinese population but remains to be further explored in the Caucasian population.

More recently, Spalinger et al [39] investigated the association between PTPN22 rs2476601 polymorphism and clinical features in 2028 IBD patients. They found that TT and CT genotypes were associated with less use of steroids and antibiotics and a lower prevalence of vitamin D and calcium deficiency in $\mathrm{CD}$, which again indicates a protective role for the $\mathrm{T}$ allele. Interestingly, the authors found that TT and CT genotypes were significantly associated with an increased use of azathioprine and anti-tumor necrosis factor antibodies in UC, suggesting more severe clinical manifestations, and therefore opposite effects on disease severity compared to CD. However, in our study, we could not identify a significantly higher risk of UC with rs2476601 after pooling data from 6971 individuals. However, given the much smaller number of patients compared to those with $\mathrm{CD}$, there may have been insufficient statistical power to detect a possible difference.

Several limitations in our study should be considered. First we could not perform a stratified analysis because of the lack of data on other ethnic groups. Most of the studies involved a Caucasian population. Gene polymorphism variation among different ethnicities was not explored. According to the study of Chen et al [22], the T allele was absent in rs2476601, suggesting that the protective effect on $\mathrm{CD}$ is exclusive to non-Asian ethnic groups. In addition, clinical subtypes of IBD, especially relating to the severity of the diseases, could not be taken into account due to insufficient data.

Nevertheless, the present meta-analysis also had several strengths. First, no publication biases were detected, indicating that the results may be unbiased. Second, a significant number of cases and controls were included in the current study by using an effective and efficient search strategy to increase the statistical power of the analysis. Third, to our knowledge, this is the first meta-analysis to assess the association between PTPN22 and IBD.

In conclusion, the present meta-analysis pooled both statistically significant and non-significant findings from individual studies to improve the statistical performance and generate a precise conclusion. The findings of this metaanalysis demonstrate that rs2476601 polymorphism is an important risk factor for CD but not for UC. However, larger and well-designed multicenter studies, particularly addressing the Asian population group, stratified by gene-gene and gene-environment interactions, are warranted to validate our findings. 


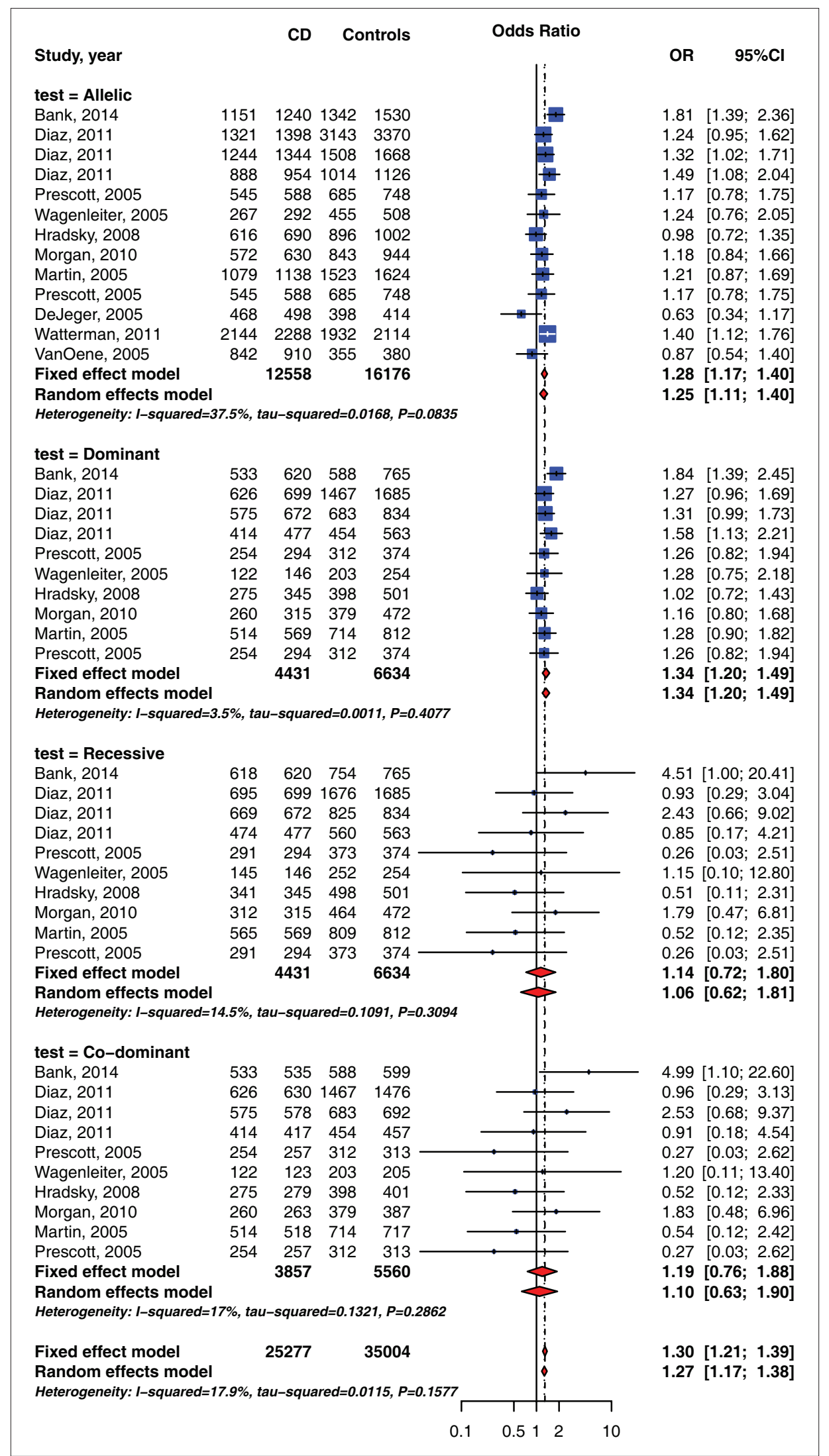

Figure 2 Risk of Crohn's disease associate with C allele, assuming an allelic, dominant, recessive, and co-dominant model CD, Crohn's disease; OR, odds ratio; 95\%CI, 95\% confidence interval 


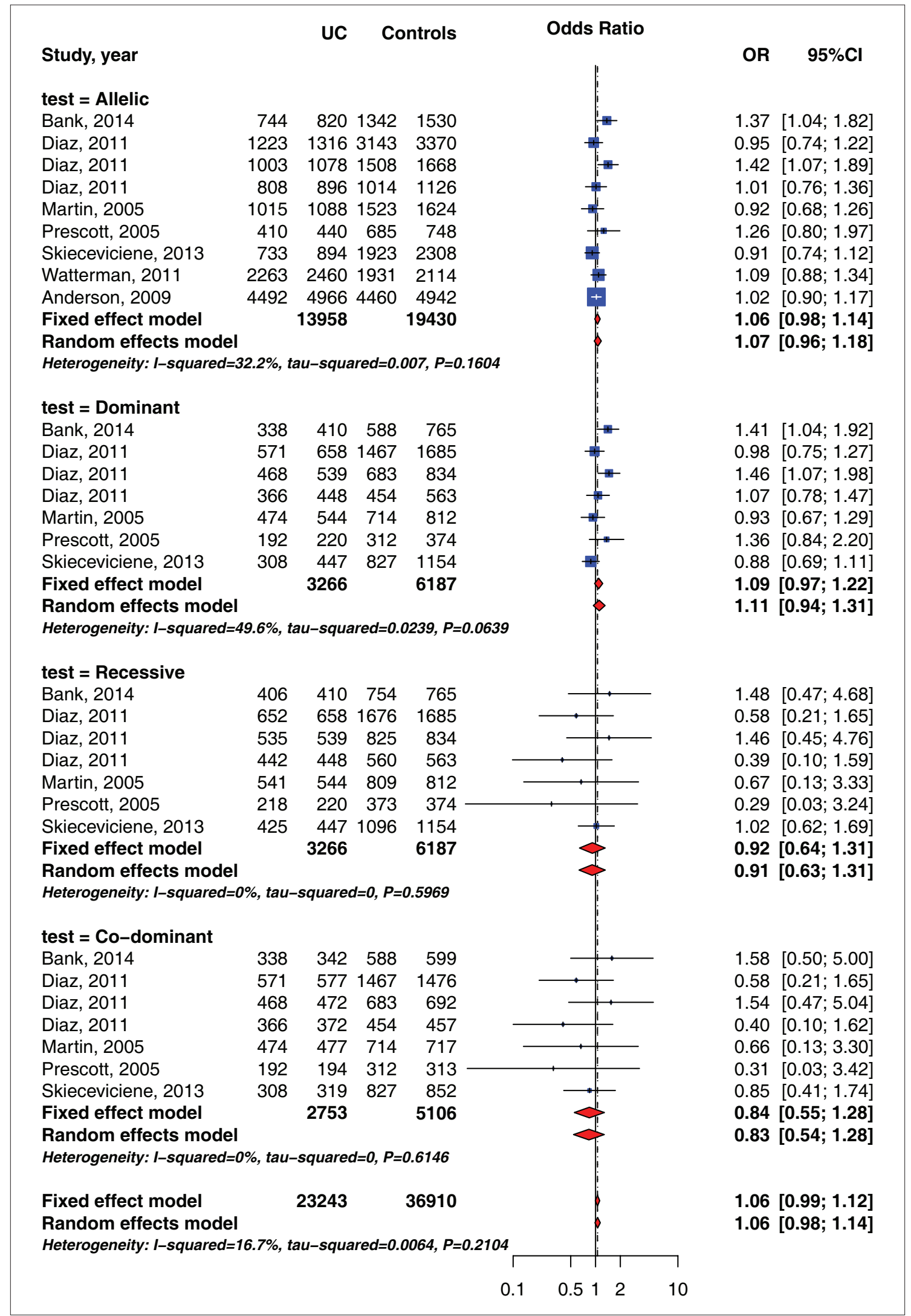

Figure 3 Risk of ulcerative colitis, assuming an allelic, dominant, recessive and co-dominant model CD, Crohn's disease; OR, odds ratio; 95\%CI, 95\% confidence interval 


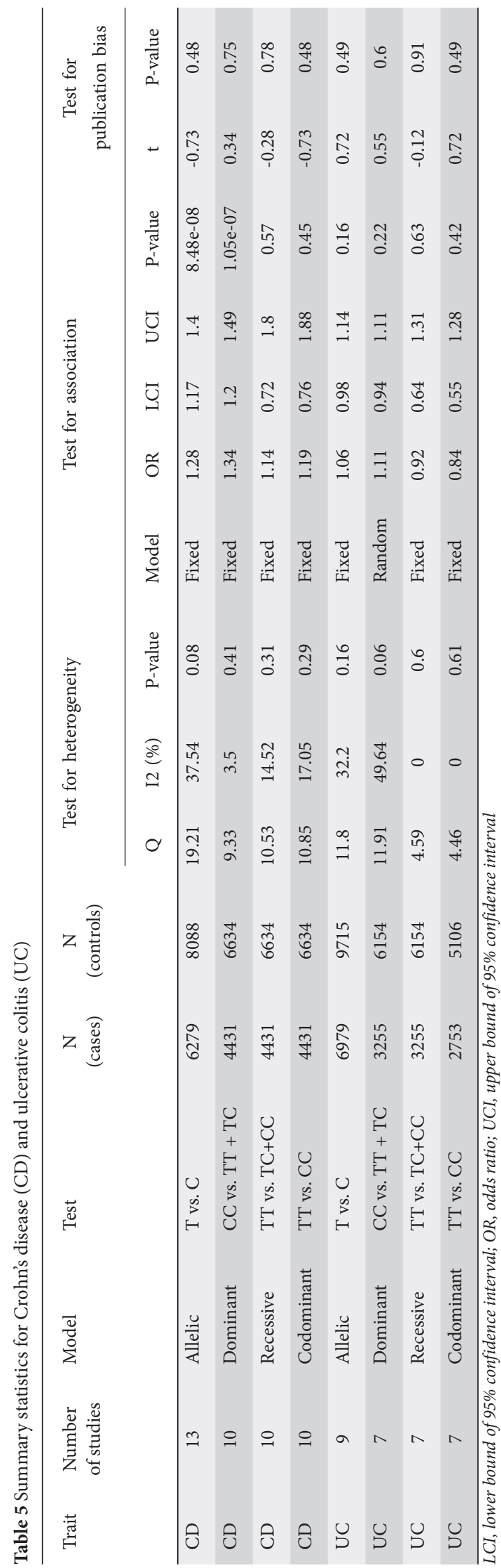

\section{Summary Box}

\section{What is already known:}

- The incidence and prevalence of inflammatory bowel diseases (IBD) are increasing over time and in different regions around the world, but their etiology remains unknown

- One of the most important non-HLA common susceptibility alleles for autoimmunity is the $1858 \mathrm{C} / \mathrm{T}$ single nucleotide polymorphism of protein tyrosine phosphatase non-receptor 22 (PTPN22) (rs2476601)

- The PTPN22 gene is located on chromosome 1p13.3-p13.1 and encodes a lymphoid-specific phosphatase

- The association between PTPN22 R620W polymorphism and IBD is inconsistent among candidate gene studies

\section{What the new findings are:}

- Individuals carrying minor allele C in rs2476601 have a greater risk of developing Crohn's disease relative to those carrying $\mathrm{T}$ allele, under an allelic and dominant model

- No significant results were found concerning ulcerative colitis, suggesting different underlying biological pathways

- No significant risk association could be identified under the recessive or co-dominant model for ulcerative colitis and Crohn's disease. Studies were not heterogeneous and no publication bias has been identified

\section{References}

1. Baumgart DC, Sandborn WJ. Crohn's disease. Lancet 2012;380:1590-1605.

2. Ordás I, Eckmann L, Talamini M, Baumgart DC, Sandborn WJ. Ulcerative colitis. Lancet 2012;380:1606-1619.

3. Molodecky NA, Soon IS, Rabi DM, et al. Increasing incidence and prevalence of the inflammatory bowel diseases with time, based on systematic review. Gastroenterology 2012;142:46-54.

4. Barrett JC, Hansoul S, Nicolae DL, et al; Wellcome Trust Case Control Consortium. Genome-wide association defines more than 30 distinct susceptibility loci for Crohn's disease. Nat Genet 2008;40:955-962.

5. Ek WE, D'Amato M', Halfvarson J. The history of genetics in inflammatory bowel disease. Ann Gastroenterol 2014;27:294-303.

6. Franke A, McGovern DP, Barrett JC, et al. Genome-wide metaanalysis increases to 71 the number of confirmed Crohn's disease susceptibility loci. Nat Genet 2010;42:1118-1125.

7. Cloutier JF, Veillette A. Cooperative inhibition of T-cell antigen receptor signaling by a complex between a kinase and a phosphatase. J Exp Med 1999;189:111-121. 
8. Burn GL, Svensson L, Sanchez-Blanco C, Saini M, Cope AP. Why is PTPN22 a good candidate susceptibility gene for autoimmune disease? FEBS Lett 2011;585:3689-3698.

9. van Oene M, Wintle RF, Liu X, et al. Association of the lymphoid tyrosine phosphatase R620W variant with rheumatoid arthritis, but not Crohn's disease, in Canadian populations. Arthritis Rheum 2005;52:1993-1998.

10. Orozco G, Sánchez E, González-Gay MA, et al. Association of a functional single-nucleotide polymorphism of PTPN22, encoding lymphoid protein phosphatase, with rheumatoid arthritis and systemic lupus erythematosus. Arthritis Rheum 2005;52:219-224.

11. Skórka A, Bednarczuk T, Bar-Andziak E, Nauman J, Ploski R. Lymphoid tyrosine phosphatase (PTPN22/LYP) variant and Graves' disease in a Polish population: association and gene dosedependent correlation with age of onset. Clin Endocrinol (Oxf) 2005;62:679-682.

12. Chelala C, Duchatelet S, Joffret ML, et al. PTPN22 R620W functional variant in type 1 diabetes and autoimmunity related traits. Diabetes 2007;56:522-526.

13. Moher D, Liberati A, Tetzlaff J, Altman DG; PRISMA Group. Preferred reporting items for systematic reviews and metaanalyses: the PRISMA statement. Int J Surg 2010;8:336-341.

14. Bowden J, Tierney JF, Copas AJ, Burdett S. Quantifying, displaying and accounting for heterogeneity in the meta-analysis of RCTs using standard and generalised Q statistics. BMC Med Res Methodol 2011;11:41.

15. Davey Smith G, Egger M. Meta-analyses of randomised controlled trials. Lancet 1997;350:1182.

16. DerSimonian R, Laird N. Meta-analysis in clinical trials. Control Clin Trials 1986;7:177-188.

17. Higgins JP, Thompson SG. Quantifying heterogeneity in a metaanalysis. Stat Med 2002;21:1539-1558.

18. Egger M, Davey Smith G, Schneider M, Minder C. Bias in metaanalysis detected by a simple, graphical test. BMJ 1997;315:629-634.

19. Diaz-Gallo LM, Espino-Paisán L, Fransen K, et al. Differential association of two PTPN22 coding variants with Crohn's disease and ulcerative colitis. Inflamm Bowel Dis 2011;17:2287-2294.

20. Latiano A, Palmieri O, Valvano MR, et al. Evaluating the role of the genetic variations of PTPN22, NFKB1, and FcGRIIIA genes in inflammatory bowel disease: a meta-analysis. Inflamm Bowel Dis 2007;13:1212-1219.

21. Sfar I, Ben Aleya W, Mouelhi L, et al. Lymphoid tyrosine phosphatase R620W variant and inflammatory bowel disease in Tunisia. World J Gastroenterol 2010;16:479-483.

22. Chen Z, Zhang H, Xia B, et al. Association of PTPN22 gene (rs2488457) polymorphism with ulcerative colitis and high levels of PTPN22 mRNA in ulcerative colitis. Int J Colorectal Dis 2013;28:1351-1358.

23. Anderson CA, Massey DC, Barrett JC, et al; Wellcome Trust Case Control Consortium. Investigation of Crohn's disease risk loci in ulcerative colitis further defines their molecular relationship. Gastroenterology 2009;136:523-529.e3.

24. Bank S, Skytt Andersen P, Burisch J, et al. Polymorphisms in the inflammatory pathway genes TLR2, TLR4, TLR9, LY96, NFKBIA, NFKB1, TNFA, TNFRSF1A, IL6R, IL10, IL23R, PTPN22, and
PPARG are associated with susceptibility of inflammatory bowel disease in a Danish cohort. PLoS One 2014;9:e98815.

25. Criswell LA, Pfeiffer KA, Lum RF, et al. Analysis of families in the multiple autoimmune disease genetics consortium (MADGC) collection: the PTPN22 $620 \mathrm{~W}$ allele associates with multiple autoimmune phenotypes. Am J Hum Genet 2005;76:561-571.

26. Jager PL De, Sawcer S, Waliszewska A, et al. Evaluating the role of the $620 \mathrm{~W}$ allele of protein tyrosine phosphatase PTPN22 in Crohn's disease and multiple sclerosis. Eur J Hum Genet 2006;14:317-321.

27. Hradsky O, Lenicek M, Dusatkova P, et al. Variants of CARD15, TNFA and PTPN22 and susceptibility to Crohn's disease in the Czech population: high frequency of the CARD15 1007fs. Tissue Antigens 2008;71:538-547.

28. Martín MC, Oliver J, Urcelay E, et al. The functional genetic variation in the PTPN22 gene has a negligible effect on the susceptibility to develop inflammatory bowel disease. Tissue Antigens 2005;66:314-317.

29. Morgan AR, Han DY, Huebner C, Lam WJ, Fraser AG, Ferguson LR. PTPN2 but not PTPN22 is associated with Crohn's disease in a New Zealand population. Tissue Antigens 2010;76:119-125.

30. Prescott NJ, Fisher SA, Onnie C, et al. A general autoimmunity gene (PTPN22) is not associated with inflammatory bowel disease in a British population. Tissue Antigens 2005;66:318-320.

31. Skieceviciene J, Kiudelis G, Ellinghaus E, et al. Replication study of ulcerative colitis risk loci in a Lithuanian-Latvian case-control sample. Inflamm Bowel Dis 2013;19:2349-2355.

32. Wagenleiter SE, Klein W, Griga T, Schmiegel W, Epplen JT, Jagiello P. A case-control study of tyrosine phosphatase (PTPN22) confirms the lack of association with Crohn's disease. Int J Immunogenet 2005;32:323-324.

33. Waterman M, Xu W, Stempak JM, et al. Distinct and overlapping genetic loci in Crohn's disease and ulcerative colitis: correlations with pathogenesis. Inflamm Bowel Dis 2011;17:1936-1942.

34. Stanford SM, Mustelin TM, Bottini N. Lymphoid tyrosine phosphatase and autoimmunity: human genetics rediscovers tyrosine phosphatases. Semin Immunopathol 2010;32:127-136.

35. Silverberg MS, Cho JH, Rioux JD, et al. Ulcerative colitis-risk loci on chromosomes 1p36 and 12q15 found by genome-wide association study. Nat Genet 2009;41:216-220.

36. Zhang J, Zahir N, Jiang Q, et al. The autoimmune diseaseassociated PTPN22 variant promotes calpain-mediated Lyp/ Pep degradation associated with lymphocyte and dendritic cell hyperresponsiveness. Nat Genet 2011;43:902-907.

37. Liu F, Liu J, Zheng TS, et al. The $-1123 \mathrm{G}>\mathrm{C}$ variant of PTPN22 gene promoter is associated with latent autoimmune diabetes in adult Chinese Hans. Cell Biochem Biophys 2012;62:273-279.

38. Viken MK, Olsson M, Flåm ST, et al. The PTPN22 promoter polymorphism $-1123 \mathrm{G}>\mathrm{C}$ association cannot be distinguished from the $1858 \mathrm{C}>\mathrm{T}$ association in a Norwegian rheumatoid arthritis material. Tissue Antigens 2007;70:190-197.

39. Spalinger MR, Zeitz J, Biedermann L, et al; Swiss IBD Cohort Study Group. Genotype-phenotype associations of the CD-associated single nucleotide polymorphism within the gene locus encoding protein tyrosine phosphatase non-receptor type 22 in patients of the Swiss IBD cohort. PLoS One 2016;11:e0160215. 